encompassing 18 out of the 30 items, to be the most theoretically meaningful factor structure. This six-factor model was affirmed by a CFA $(\mathrm{CFI}=0.94$, TLI $=0.92$, RMSEA $=0.036)$. These factors were, namely, 'the image of psychiatrists', 'psychiatric patients and mental illness', 'efficacy of treatment', 'psychiatric teaching', 'career choice', and 'psychiatry as an evidence-based discipline'. This six-factor solution was invariant across gender. 'The image of psychiatrists' appeared to be the most salient factor, and formed the most consistent subscale $(\omega=0.71)$. The internal consistencies of the other subscales were modest $(\omega=0.55-0.67)$. The overall 18 -item scale showed good internal consistency $(\omega=0.78)$.

Conclusion. Our findings provide evidence of a multidimensional structure in medical students' attitudes towards psychiatry, endorsing six meaningful subscales of the ATP-30. Future researchers and educators can utilize these subscales in identifying specific attitudinal domains which are more closely associated with students' future choice of a career in psychiatry, and also in identifying specific areas where attitudes are more stigmatized, so that appropriate interventions can be incorporated into the undergraduate psychiatric curriculum.

\section{Virtual clinical examinations: are they the new 'normal'?}

Mahmoud Barakat ${ }^{1 \star}$, Hannah Slevin ${ }^{1}$, Reshmi Nijjar ${ }^{2}$,

Kavitha Ramamurthy ${ }^{1}$, Latha Hackett ${ }^{3}$

and Dushyanthan Mahadevan ${ }^{3}$

${ }^{1}$ Manchester Foundation Trust; ${ }^{2}$ East Lancashire Hospitals NHS

Trust and ${ }^{3}$ Health Education England North West

${ }^{*}$ Corresponding author.

doi: 10.1192/bjo.2021.361

Aims. The North West School of Psychiatry run a yearly structured clinical examination to help Core Psychiatry Trainees develop their training competencies and prepare for the MRCPsych Clinical Assessment of Skills and Competencies (CASC). Historically, this has been face-to-face with logistical difficulties, high cost, low trainee uptake and challenging in recruiting examiners. Following the COVID-19 pandemic and the subsequent shift to virtual consultations and examinations, the team implemented an innovative virtual Skills test. The main aims were to improve the test's quality and the trainee uptake, adapt the test delivery to a Health Education England (HEE) online platform, and establish cost-effectiveness in the post-COVID world.

Method. A working group was formed to develop the Skills test, and in May 2019, the test was delivered face-to-face, implementing 5 cycles of 8 stations over 3 days. The same group adapted the test for online delivery, and in August 2020, 3 cycles of 8 stations were delivered. Feedback was collected, with adaptations made for a second Skills Test in December 2020.

Result. $96.4 \%$ of trainees rated their overall experience and the test organisation in the 2019 test as excellent or good $(82.1 \%$ and 85.7 excellent, respectively). $93.5 \%$ of examiners rated their overall experience and the test organisation as excellent or good (45.1\% excellent for both). In the August test, $95.8 \%$ of trainees rated their overall experience as excellent or good (58.3\% excellent). $100 \%$ of trainees rated the test organisation and the online format as excellent or good $(70.8 \%$ and $50 \%$ excellent, respectively). Although $100 \%$ of examiners rated the overall experience, the test organisation and online format as excellent or good, some felt the stations were not long enough to allow for technical issues. In the December test, higher proportions of trainees rated the overall experience $(80 \%)$, organisation of the test $(80 \%)$ and online format $(70 \%)$ as excellent.

Conclusion. The virtual test is shown to be a viable and successful alternative to the face-to-face test in preparing trainees for their CASC, and trainees felt it was excellent preparation for the new online CASC format. It had some clear advantages, such as saving on consumables, reducing the financial costs of running the test, improving the test quality, and increasing the trainee uptake. It is more eco-friendly and reduces fuel emission, raising the question of how the test should be delivered after the COVID-19 pandemic.

Attitude \& belief towards mental illness and psychiatry as a faculty among medical students at International Medical University, Malaysia

Debakanta Behera ${ }^{1 \star}$ and Ji Yen $\mathrm{Ku}^{2}$

${ }^{1}$ NWBH NHS Foundation Trust and ${ }^{2} 5$ th year medical undergraduate, University of Liverpool

${ }^{\star}$ Corresponding author.

doi: 10.1192/bjo.2021.362

Aims. Third year Medical students from the International Medical University, Malaysia were assessed regarding their commonly held attitudes and beliefs for the mental illness in general as well as with respect to psychiatry as a faculty through a survey monkey based survey,

Background. Commonly held perceptions and prejudices often can be overcame by education and early exposure to facts which also holds true with medical students and their attitude as well as expectations to psychiatry. Ever growing awareness regarding the Mental illness has helped but is unable to complete address the stigma and prejudices associated with it. Also Early exposure to psychiatry in medical education can provide a positive experience to medical students including germinating an interest in psychiatry as a career choice among the students.

Method. 42 students of 3rd and 4th year medical school from International Medical University, Malaysia, some without any exposure to psychiatry, were participated in a survey created on a cloud based online survey link and responded to a questionnaire about the attitude and belief towards mental Illness as well as Psychiatry as a career choice. The results were analysed and data interpreted.

Result. Most students (85\%) though agreed that psychiatry is a rapidly expanding frontier of medicine sadly only $20 \%$ stated that it would be one of the top three career choice. Just under the $50 \%$ of the students stated that the psychiatric patients are more likely to harm others. About 95\% felt that psychiatric consultations of patients with medical and surgical health problems would be helpful and 90\% students shared that they would not feel embarrassed about someone from their family if diagnosed with mental illness.

Conclusion. Psychiatric exposure in medical education has been recognised as inadequate in general and often exposing medical students to psychiatry early helps improving the stigma and prejudices associated with mental illness. It will also give them sufficient exposure to assess the illness holistically keeping mental health in mind while treating physically ill people and also may inspire them to choose psychiatry as a career choice in a rapidly developing and conservative country such as Malaysia where mental health services are largely inadequate and is the second biggest health issue. 